\title{
Before and after spectra: Enhanced chemical speciation below former detection limits
}

\author{
S NEHZATI $^{1 *}$, NV DOLGOVA ${ }^{2}$, D SOKARAS ${ }^{3}$, T KROLL $^{3}$, \\ IJ PICKERING ${ }^{2}$ AND GN GEORGE ${ }^{2}$
}

${ }^{1}$ MAX IV, Lund University, Lund, 224 84, Sweden

(correspondence: susan.nehzati@maxiv.lu.se)

${ }^{2}$ Geological Sciences, University of Saskatchewan, Saskatoon, SK, Canada S7N 5E2

${ }^{3}$ Stanford Linear Accelerator Center, Menlo Park, CA, 94025, USA

\section{Background}

Mercury compounds can be some of the most potent toxicants humans are exposed to as a result of both environmental and anthropogenic activity. Precise chemical species identification is crucial in understanding the mechanistic aspects of toxicity, and accordingly, strengthening strategies designed to mitigate human exposure and remediate hazardous materials. Recent advances in chemical analysis using synchrotron X-ray methods allow distinct chemical form identification at naturally low levels. As a result, this has provided answers to challenging questions in various health and environmental systems [1][2].

\section{Discussion of Results}

Emission spectroscopic techniques have emerged as providing great opportunity in chemical species identification of heavy elements [3][4]. With high-energy resolution fluorescence detected X-ray absorption spectroscopy (HERFD-XAS), structural elemental analysis at concentrations as low as $100 \mathrm{nM}$ is achievable, revealing rich spectroscopic detail and permitting greater distinction between structurally similar compounds. Case studies investigating structural characterization of a number of mercury compounds will be discussed, together with selenium compounds, as the toxicity of mercury is closely connected to the biochemistry of selenium. Examples of mercury and selenium found in plant and mammalian tissue will be presented. Results indicate unexplored spectroscopic detail thus improving the fingerprinting capabilities of XAS. Experiments are ongoing as these methods aid in unravelling answers to a number of toxic mechanisms occuring in the world around us.

[1] Manceau et al. (2016) ES\&T 50, 10721-10729.

[2] Herzog et al. (2020) Biogeosciences 17, 331-344.

[3] James et al. (2020) ES\&T 54, 2726-2733.

[4] Vogel et al. (2020) J Hazard Mater 382, 121100. 